\title{
Pyrexia of unknown origin 90 years on: a paradigm of modern clinical medicine
}

\author{
Michael Brown
}

\section{Correspondence to} Dr Michael Brown, Hospital for Tropical Diseases, University College London Hospitals NHS Trust, Maple House, Tottenham Court Road, London WC1E 6BU, UK mike.brown@uclh.nhs.uk

Received 27 May 2015 Revised 15 September 2015 Accepted 28 September 2015 Published Online First 21 October 2015

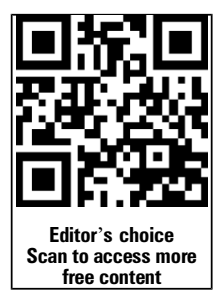

CrossMark

To cite: Brown M. Postgrad Med J 2015;91:665-669.

\section{ABSTRACT}

In 1925, Sir Thomas Horder, a leading physician of his day, gave a lecture, published in this journal, entitled 'Some cases of pyrexia without physical signs'. The paper highlighted what was already a familiar clinical presentation "which taxes our resources to the utmost". Fast-forward through 90 years of careful clinical description, technological innovation in diagnosis and treatment, emergent infections, novel diagnoses, demographic shifts, and radical changes in the health economy. Sir Thomas would find certain aspects familiar, and others revolutionary, in the differential diagnosis and management of the 21st century patient with pyrexia of unknown origin (PUO). Within high-income settings, the proportion of cases due to infection has declined, albeit unevenly. The era of untreated HIV, and the consequences of iatrogenic intervention and immunosuppression, led to Durack and Street's subclassification of the condition in the early 1990s into classic, nosocomial, neutropenic and HIV-associated PUO. Shifts towards ambulatory care have driven a change in the definition of many diseases. An era of observant clinicians, who lent their names to eponymous syndromes, followed by meticulous serological, genetic and clinicopathological correlation, generated a battery of diagnoses that, along with malignancy, form a large proportion of diagnoses in more recent clinical care. In the current era, universal access to cross-sectional imaging and an infinite array of laboratory tests has undermined the attention paid to history and examination. In some areas of the clinical assessment, such as assessing the fever pattern, this shift is supported by research evidence. The issues that need to be addressed in the next 90 years of technological innovation, information sharing and health service transformation are likely to include: transcriptomic approaches to diagnosis; the place of positron emission tomography (PET) in the diagnostic pathway; the optimal management of high ferritin states; and the most costeffective diagnostic environment, in the face of this era of specialisation and fragmentation of care. In the meantime, this review covers some important early $21 \mathrm{st}$ century lessons to be shared in avoiding diagnostic pitfalls and choosing empirical therapy.

\section{INTRODUCTION}

Imagine Sir Thomas Horder, whose lecture on 'Some cases of pyrexia without physical signs' was published in this journal in 1925, transported to a 21st century London hospital, an observer in the clinic (with honorary contract and criminal disclosure check complete). ${ }^{1}$ What aspects of the cases described here would most perplex Sir Thomas? Where would he find comfort in familiarity (or disappointment in lack of progress)? The evolution and revolutions in the practice of medicine, in highincome settings, over the last 90 years, are aptly illustrated by, and refracted through, the diagnosis and management of pyrexia of unknown origin (PUO).

\section{DECLINE IN INFECTIOUS AETIOLOGY}

Neither of the cases described in boxes 1 and 2 were due to an infection (as far as current understanding of large vessel vasculitis suggests). In contrast to case series from the first half of the 20th century, the last few decades have seen a decline in infectious causes and an increase in inflammatory and malignant aetiologies. By the time Petersdorf established his seminal definition of fever of unknown origin, he found an infectious cause in only a third of cases studied (the research was undertaken in 1952-1957), approximately half the rate seen in studies he cited from the $1930 \mathrm{~s} ;{ }^{2}$ subsequent prospective case series have had generally lower infection prevalences. ${ }^{3-5}$

Reductions in the incidence of chronic suppurative and other bacterial infections can be attributed to the advent and widespread availability of antibiotics since the first use of sulphonamides in the 1930s and penicillin in the 1940s, and to the reduction in poverty-related infectious diseases in higher income countries, such as enteric fever and tuberculosis (TB). However, the decline has not been relentless. The 30-year era of untreated advanced HIV is surely nearing its end but has left a legacy of opportunistic infectious diagnoses (cytomegalovirus, atypical mycobacterial infection, histoplasmosis) in the differential diagnosis of the young febrile immunosuppressed patient, and for this patient group surplanted Occam's razor (diagnostic parsimony) with Hickam's dictum ('a man can have as many diseases as he damn well pleases'). Simultaneousy, huge migration shifts from settings of high infectious disease prevalence, especially over the last 50 years, have driven up, for example, TB incidence rates in European and US settings, with demonstrable delay in recognition by an era of medical professionals for whom the disease had become a rarity. The need for a travel history, an understanding of the epidemiology of infectious conditions in the country of origin, and recognition of the concept of latency, has never been greater.

Durack's 1991 classification of classic, nosocomial, neutropenic and HIV-associated PUO has been an illustrative paradigm reflecting some of these epidemiological drivers and remains a critical framework for a focussed diagnostic approach. ${ }^{6}$

\section{ANTIMICROBIAL RESISTANCE}

Less a diagnostic than a therapeutic challenge is the emergence of antimicrobial resistance-mainly an 


\section{Box 1 Case 1}

A 51-year-old journalist presented to the infectious diseases clinic with a 3-week history of fevers, drenching night sweats, lassitude and a dry cough. He gave a longer approximately 3-month history of headaches and upper arm and thigh myalgia. Over this 3-month period he had two transient temporary visual obscurations. Examination was normal, apart from a temperature of $38^{\circ} \mathrm{C}$. There were no murmurs, and no temporal artery tenderness; fundoscopy was normal. Dipstick of urine revealed ++ protein and ++ blood. Erythrocyte sedimentation rate was $110 \mathrm{~mm} / \mathrm{h}$, and C-reactive protein was $178 \mathrm{mg} / \mathrm{L}$. Biochemistry (including creatine kinase) was otherwise normal. There was a mild neutrophilia. Chest radiograph was normal, as was an abdominal ultrasound. HIV serology was negative. Two sets of blood cultures were negative. A positron emission tomography (PET)/CT scan performed $48 \mathrm{~h}$ later demonstrated diffusely increased uptake in the medium and large vessels (carotids, subclavian, aorta and proximal common iliac arteries). A diagnosis of large vessel vasculitis was made, and oral prednisolone was started on day 3 with rapid clinical and biochemical response.

imported (and iatrogenic) threat that could in future reverse the decline in the infection-attributable proportion of fever in the clinic. Currently, at least in high-income settings, examples of persistent fever attributable to iatrogenic antimicrobial resistance are arguably limited to multidrug-resistant TB, enteric fever and gram-negative neutropenic sepsis. But evolving resistance in, for example, sexually transmitted infections, may generate future examples.

\section{TECHNOLOGICAL INNOVATION IN IMAGING AND LABORATORY DIAGNOSTICS}

The shift towards non-infectious causes can be explained partly by other demographic shifts, not least the ageing population, among whom malignant and inflammatory causes predominate. But an analysis of such trends needs above all to take account of the technological advances in diagnosis, and hence in disease classification, that have so transformed modern medicine. A glance at the contents page of a contemporary medical textbook from Sir Thomas's era, such as Osler's Principles and Practice of Medicine (the 10th Edition was edited by McCrae and published in the early 1920s), demonstrates the near total absence of non-

\section{Box 2 Case 2}

A 27-year-old Turkish Kurdish man was referred to the infectious diseases clinic with a 6-year history of increasingly severe attacks characterised by peritonitic abdominal pain, myalgia and fever, lasting 1-2 days every 1-4 weeks. During an episode his C-reactive protein and neutrophil count were elevated, but thereafter settled spontaneously. His pyrin (MEFV) gene was sequenced and revealed a compound heterozygote genotype, with two recognised pathogenic mutations in exon 10 encoding the pyrin variants M680I and V726A. A diagnosis of familial Mediterranean fever was thus confirmed, and his condition effectively managed with daily prophylactic colchicine. infectious inflammatory conditions, and to some extent of malignancies. By contrast, in a recent PUO case series, the majority of non-infectious inflammatory and miscellaneous diagnoses, constituting approximately a third of the final diagnoses, were conditions which were not recognised in $1925 .^{5}$ Entities such as drug-induced fever, adult-onset Still's disease and periodic fever syndromes, are now common diagnoses among cryptic febrile patients in the modern hospital setting.

In the early 20th century, the diagnostic approach was one of careful case description, close attention to the fever pattern, and clinic-pathological correlation. This provided the gold standard for future developments but has been overwhelmed by the focus over the last 40 years on imaging and laboratory diagnostics. The first CT scanner was installed in Wimbledon, where Hounsfield scanned the first patient in 1971. Since then we have seen the introduction and habitual use of CT, MRI, echocardiography, radiolabelled white cell scans and, most recently, positron emission tomography (PET) scans in the investigation of the febrile patient. In the laboratory, the limitations of microscopy and culture techniques were addressed by serology and subsequently molecular genetics-the latter through restriction fragment length polymorphism and then PCR techniques, and most recently next generation sequencing (NGS) for high throughput identification of novel pathogens, which may revolutionise clinical diagnostics in the next decade. $^{7-10}$

Analysis of NGS data requires massive computational power, currently delivered through thousands of networked home computers - the latest in a history of internet use for PUO diagnosis. Earlier examples were celebrated in publications of cases where Google delivered the diagnosis. ${ }^{11}$

The complex network of inflammatory/metabolic responses that characterise febrile states, teased out and characterised by transcriptional profiling, provides another avenue for categorising the patient-will this be another nail in the coffin of bedside examination? ${ }^{12}$ Is that something to mourn, or evidence that, like relative bradycardia and the fever pattern, carefully described clinical phenomena do not match the capacity of modern imaging and laboratory tools to define and stratify illnesses. $^{13} 14$

\section{HEALTH SYSTEMS-GENERALISM OR SPECIALTY CARE?}

Which leads to the other area in which the approach to the diagnosis of PUO has been transformed. Sir Thomas Horder would probably recognise few of the characteristics of the present day UK health system. The first major change was the introduction of the National Health Service (in the face of opposition by Horder among other leaders of the medical profession) which transformed access to care and the doctorpatient relationship. Arguably the second major change has been the drive to specialisation. The proliferation of diagnoses (fewer than 400 in the International Classification of Diseases V.3 (ICD-3), which was published in 1920, compared with 7080000 in ICD-10), as well as the costs and complexities of diagnosis and therapy, has compelled this diversification of expertise. Most specialties have their organ-specific causes of PUO, and thus have a contribution to make to the assessment of the undiagnosed febrile patient. But there are patients, especially in the ambulatory setting, who fall through the fracture lines between organ-based specialties. General medicine, as a discipline, survives almost as a retort to superspecialisation, with advocacy from the Royal College of Physicians albeit prompted largely by the rising burden of age and co-morbidity in modern medicine. $^{15}$ 
Infectious diseases were so pervasive across medical disciplines that a specialty could not have been contemplated until the latter part of the 20th century, when emerging infections, drug resistance and the drive for specialisation re-created the need. Now PUO is a favoured clinical conundrum within the specialty and our expertise and focussed approach to investigation are valued. Of relevance has been clinical and academic experience in, and links with infection specialists in, travel destinations and the countries of origin of migrants, where the epidemiology of infectious disease is highly relevant to focused investigation and treatment in the UK. Access to infectious diseases expertise remains patchy across the UK, but core medical and specialist training, co-specialisation with general medicine or microbiology, and other factors are helping to fill the gaps. The success of the specialty in delivering better care for the PUO patient is difficult to measure, and dependent on many local health system variables such as timely access to diagnostics, a dedicated specialty bed-base, and above all multidisciplinary communication with mutual trust and respect.

\section{EVOLVING CASE DEFINITIONS}

The case definition for PUO has evolved over the decadesfrom Petersdorf's 'illness of more than three weeks' duration, with fever $>101^{\circ} \mathrm{F}$ on several occasions, and diagnosis uncertain after one week in hospital" to Durack's "at least three outpatient visits or at least 3 days in hospital" and more recently, Knockaert and Vanderschueren's "no diagnosis after an initial diagnostic evaluation" (with a specified minimum investigation set), and their inclusion of patients who do not meet the fever threshold but have high circulating inflammatory markers. ${ }^{16} \mathrm{~A}$ standardised case definition is clearly a prerequisite to systematic evaluation of the literature, and the variance in population, specialism, exclusions and other factors between studies is perhaps the primary reason that high quality evidence-based approaches to PUO are sparse. ${ }^{17}$ And thus why overviews on the subject (the present article included) tend to find more value in highlighting diagnostic pitfalls- the 'dos and don'ts'in the investigation of what is a heterogeneous and (by necessity of local health system referral routes) a locally defined clinical entity. ${ }^{18}$

\section{DIAGNOSTIC PITFALLS}

Sir Thomas Horder highlighted some key elements of his diagnostic arsenal that were, in his era, underused or misunderstood. Most were clinical examination findings and paradigms elicited by careful observation. They remain valuable-perhaps none more so than his recommendation to repeat the examination and give attention to the classic refuge sites for pus (liver, bone, heart). What might constitute a modern specialist's dogma on diagnosis and empirical therapy of PUO?

In 2015, the role of the examination is necessarily less important when cross-sectional imaging is so pervasive a part of management. It is difficult to imagine how a careful clinical history, by contrast, could ever lose its key role in negotiating a rational route through the plethora of serological assays listed on the request form of a 21st century hospital laboratory. The principle to remember here is one of Bayesian probability. A test with $99.9 \%$ specificity will generate more false positives than false negatives if used in a patient for whom the diagnosis in question is very unlikely, for example, where there is less than one chance in a 1000 (examples would be Lyme serology as a diagnostic test for PUO where there is no history of exposure). This is also an issue for molecular techniques in immunocompetent patients, in whom the yield is low and the potential for false positives is high. ${ }^{19}$ Such tests should be requested with caution.

TB remains an important diagnosis in this patient group, and the obvious clues (albeit often a distant travel history) should prompt investigation. There are, however, common pitfalls here. The old textbook recommendation of testing early morning urines seems to be a difficult maxim to shake off. Diagnostic utility is very low in the HIV-negative patient, in whom urinary tract $\mathrm{TB}$ is rare and anyway associated with obvious imaging abnormalities, and in whom the time to positivity of cultures is too long to play a role in the diagnostic pathway. More concerning is the indiscriminate use of immunological assays, previously the tuberculin skin test but recently the interferon- $\gamma$ release assay (IGRA), in the diagnosis of TB as a cause of PUO. The literature has been heterogeneous but in general highlights the limited sensitivity, and specificity, of IGRA for the diagnosis of active TB. ${ }^{20}{ }^{21}$ All infectious diseases specialists have experience of the patient with active TB and a negative IGRA, and yet many still find themselves using the test in some patients-albeit with open eyes-its role perhaps most useful in tilting the differential in the patient without risk factors for exposure, but with granulomatous pathology or inaccessible (eg, brain) pathology on imaging. The tuberculin skin test, by contrast, perhaps has more use in evaluating a potential diagnosis of sarcoid (by demonstrating anergy) than TB. ${ }^{22}$

Ferritin is a favoured test in the investigation of PUO, in supporting a diagnosis on the macrophage activation syndrome spectrum-for example, adult onset Still's disease or haemophagocytic syndrome. Specificity is, however, limited, with sufficient overlap with infectious and other aetiologies to render it far from a diagnostic test. ${ }^{23}$

Bone marrow examination is useful-albeit pick-up from culture minimal (except in the HIV-infected population), compared with the yield from histopathological analysis (in particular haematological malignancy or haemophagocytosis). There are predictive clinical and laboratory features for bone marrow yield but in the PUO population it is questionable how clinically valuable these predictors are. It is useful to know that steroids before bone marrow biopsy do not reduce the yield. Visceral leishmaniasis is one of the few infectious diagnoses made by this method, but might be suspected by other clinical features, and in the immunocompetent the serology will be positive. Peripheral flow cytometry has been advocated for the diagnosis of occult haematological malignancy, but the additional yield is low. 2425

PET/CT has a high diagnostic yield in PUO but the literature belies the complexity of its real place in investigation. ${ }^{26}$ Few diagnoses are detected on PET that are not visualised on standard cross-sectional CT imaging; large vessel vasculitis is arguably the only one of relevance in this patient group. Identifying clinical features (as in our introductory case) that predict this diagnosis might further define the use of this modality in the diagnostic pathway. Use in patients with contraindications to contrast, or with hyperglycaemia, or for targeting a biopsy in the patient with multifocal lymphadenopathy, are other situations where PET may have a defined role. Currently it is perhaps overused in centres with access, although this may change under cost pressures. ${ }^{27} 28$

Rheumatologists, as specialists with expertise in a range of systemic inflammatory disorders, have an obvious and valuable role in the assessment of many patients with PUO. Positive autoantibodies need interpretation in the context of the patient's clinical features, and seronegative connective tissue disorders are well recognised. However, a common pitfall is to equate no 
evidence of a rheumatological condition with a situation where it is unlikely to be a steroid-responsive disease, with consequent delay in the treatment of a patient for whom infection and malignancy have been adequately excluded. The macrophage activation syndromes are a case in point, for which delay in treatment appears to be associated with poor outcome. The infectious diseases opinion is important in drawing a line under further microbiological or serological investigations or presumptive treatment and advocating for, or indeed initiating, corticosteroids.

\section{EMPIRICAL THERAPY}

Presumptive antibiotic treatment usually features in the patient's pathway through diagnosis and recovery. The classic laboratory features of adult onset Still's disease include neutrophilia and a high C-reactive protein, and it is a brave clinician who does not initiate some broad-spectrum antibiotic at least for the first few days. Presumptive specific anti-tuberculous therapy (eg, isoniazid and pyrazinamide) has rightly gone out of fashion in an era of isoniazid drug resistance and better diagnostic provision for extrapulmonary TB. Suspected enteric fever, if the history supports the diagnosis, may be reasonably approached with presumptive therapy given the imperfect diagnostic yield of the culture tests (serological tests have no utility in high-income settings). But this is an example where drug resistance needs to be taken into account. Presumptive treatment with, for example, ciprofloxacin is not indicated given the high prevalence of decreased ciprofloxacin susceptibility among confirmed Salmonella typhi and S. paratyphi isolates.

Undifferentiated fever is an important clinical entity in the tropical clinic, and in some forms has acquired the tongue-in-cheek term 'doxycycline deficiency disease'. ${ }^{29}$ The turnaround time for rickettsia, coxiella and leptospirosis diagnostics can be long, and at least for some of these conditions, treatment with doxycycline is associated with rapid defervescence (and is thus a useful diagnostic test pending laboratory confirmation) and prevention of complications.

As mentioned, there has long been a role for anti-inflammatory therapies in the empirical management of the patient with non-infectious inflammatory states, such as macrophage activation syndromes. Currently, initial treatment (eg, for adult onset Still's disease) is with corticosteroids. The literature on the use of biologic therapies such as interleukin-1 (IL-1) antagonists (such as anakinra) is evolving, and future studies might define a cohort of patients for whom such drugs might be used empirically at an earlier stage of the disease. ${ }^{30}$

\section{CONCLUSIONS}

What will be the future for patients with PUO in another 90 years? As discussed, socio-political upheaval and consequent migration, and unrelenting changes in the structure of health systems, will continue to be important factors influencing the epidemiology and approach to management. Within this context, biomedical research will yield new tools to address the differential diagnosis. These will include sensitive imaging modalities, improved access to and interpretation of host/pathogen genome and transcriptome sequencing, and clinical trial evidence to support newer biological therapies as initial presumptive treatment. Without doubt, however, misdirected laboratory investigations, poorly co-ordinated approaches to care and the sheer variety of clinical presentation will continue to define the limitations of standardised approaches to management. Sir Thomas Horder's successors in the next century will have much on which to opine.

\section{Main messages}

- Pyrexia of unknown origin (PUO) is a recognisable clinical entity, with significant diagnostic overlap with inflammation of unknown origin; case definitions evolve with the routine availability of tests and with the health economy.

- Infectious causes of PUO have declined since the beginning of the antibiotic era-at least in high-income settings - but HIV, immigration and probably antimicrobial resistance have been important, dynamic risk factors for infection as an attributable cause.

- New diagnostic modalities, especially DNA amplification, host genetic sequencing and nuclear medicine techniques, have lent precision to the diagnostic process.

- Tests—including imaging — need to be used with an understanding of their limitations.

- Presumptive therapy has a defined role-there is less use of specific tuberculosis therapy now, and a specific role for doxycycline; clinical acumen and leadership is needed for timely trials of corticosteroids.

\section{Current research questions}

- What is the place of positron emission tomography in the diagnostic pathway of pyrexia of unknown origin?

- What is the optimal management of high ferritin states: the use of IL-1 receptor antagonists, rituximab, steroids?

- What environment (hospital vs ambulant, infectious diseases specialty vs other, bedside review vs remote liaison) is associated with the most cost-effective approach to diagnosis?

- Can the transcriptome differentiate aetiologies in the investigation of pyrexia of unknown origin? And can better cytokine assays be operationalised for differentiating active from latent disease with organisms such as tuberculosis?

Competing interests None declared.

Provenance and peer review Commissioned; externally peer reviewed.

\section{REFERENCES}

1 Horder T. Some cases of pyrexia without physical signs. Postgrad Med J 1925;1:17-21.

2 Petersdorf RG, Beeson PB. Fever of unexplained origin: report on 100 cases. Medicine (Baltimore) 1961;40:1-30.

3 Bleeker-Rovers CP, Vos FJ, de Kleijn EMHA, et al. A prospective multicenter study on fever of unknown origin: the yield of a structured diagnostic protocol. Medicine (Baltimore) 2007;86:26-38.

4 De Kleijn EM, Vandenbroucke JP, van der Meer JW. Fever of unknown origin (FUO). I. A prospective multicenter study of 167 patients with FUO, using fixed epidemiologic entry criteria. The Netherlands FUO Study Group. Medicine (Baltimore) 1997;76:392-400.

5 Vanderschueren S, Knockaert D, Adriaenssens T, et al. From prolonged febrile illness to fever of unknown origin: the challenge continues. Arch Intern Med 2003; 163:1033-41.

6 Durack DT, Street AC. Fever of unknown origin--reexamined and redefined. Curr Clin Top Infect Dis 1991;11:35-51.

7 Lecuit $M$, Eloit $M$. The diagnosis of infectious diseases by whole genome next generation sequencing: a new era is opening. Front Cell Infect Microbiol 2014;4:25.

8 Naccache SN, Federman S, Veeraraghavan N, et al. A cloud-compatible bioinformatics pipeline for ultrarapid pathogen identification from next-generation sequencing of clinical samples. Genome Res 2014;24:1180-92. 
9 Naccache SN, Peggs KS, Mattes FM, et al. Diagnosis of neuroinvasive astrovirus infection in an immunocompromised adult with encephalitis by unbiased next-generation sequencing. Clin Infect Dis 2015;60:919-23.

10 Brown JR, Morfopoulou S, Hubb J, et al. Astrovirus VA1/HMO-C: an increasingly recognized neurotropic pathogen in immunocompromised patients. Clin Infect Dis 2015;60:881-8.

11 Tang $\mathrm{H}, \mathrm{Ng}$ JHK. Googling for a diagnosis--use of Google as a diagnostic aid: internet based study. BMJ 2006:333:1143-5.

12 Nemeth J, Winkler H-M, Zwick RH, et al. Peripheral T cell cytokine responses for diagnosis of active tuberculosis. PLOS ONE 2012;7:e35290.

13 Davis TM, Makepeace AE, Dallimore EA, et al. Relative bradycardia is not a feature of enteric fever in children. Clin Infect Dis 1999:28:582-6.

14 Mackowiak PA, LeMaistre CF. Drug fever: a critical appraisal of conventional concepts. An analysis of 51 episodes in two Dallas hospitals and 97 episodes reported in the English literature. Ann Intern Med 1987;106:728-33.

15 Royal College of General Practitioners and the Health Foundation. RCP comment on Commission on Generalism report. Guiding Patients through Complexity: Modern Medical Generalism. [Internet]. London, 2011 (cited 25 May 2015). https://www. rcplondon.ac.uk/press-releases/rcp-comment-commission-generalism-report-guidingpatients-through-complexity-modern-0

16 Vanderschueren S, Del Biondo E, Ruttens D, et al. Inflammation of unknown origin versus fever of unknown origin: two of a kind. Eur J Intern Med 2009;20:415-18.

17 Mourad O, Palda V, Detsky AS. A comprehensive evidence-based approach to fever of unknown origin. Arch Intern Med 2003;163:545-51.

18 Vanderschueren S, Knockaert D. Tackling fever and inflammation of unknown origin: the do's and don'ts. Acta Clin Belg 2014;69:412-17.

19 Robine A, Hot A, Maucort-Boulch D, et al. Fever of unknown origin in the 2000s: evaluation of 103 cases over eleven years. Presse Méd 2014;43:e233-40.
20 Winqvist N, Björkman P, Norén A, et al. Use of a T cell interferon gamma release assay in the investigation for suspected active tuberculosis in a low prevalence area. BMC Infect Dis 2009;9:105.

21 Hinks TSC, Dosanjh DPS, Innes JA, et al. Frequencies of region of difference 1 antigen-specific but not purified protein derivative-specific gamma interferon-secreting T cells correlate with the presence of tuberculosis disease but do not distinguish recent from remote latent infections. Infect Immun 2009;77:5486-95.

22 Vyas S, Thangakunam B, Gupta R, et al. Interferon gamma release assay and tuberculin skin test positivity in sarcoidosis. Lung India 2015;32:91-2.

23 Mehta B, Efthimiou P. Ferritin in adult-onset Still's disease: just a useful innocent bystander? Int J Inflamm 2012;2012:298405.

24 Ben-Baruch S, Canaani J, Braunstein R, et al. Predictive parameters for a diagnostic bone marrow biopsy specimen in the work-up of fever of unknown origin. Mayo Clin Proc 2012;87:136-42.

25 Hot A, Jaisson I, Girard C, et al. Yield of bone marrow examination in diagnosing the source of fever of unknown origin. Arch Intern Med 2009;169:2018-23.

26 Dong M, Zhao K, Liu Z, et al. A meta-analysis of the value of fluorodeoxyglucose-PET/PET-CT in the evaluation of fever of unknown origin. Eur J Radiol 2011;80:834-44.

27 Bharucha T, Brown M. Use of CT-PET imaging investigation of pyrexia of unknown origin. Br J Hosp Med (Lond) 2013;74:654.

28 Meller J, Sahlmann CO, Gürocak 0, et al. FDG-PET in patients with fever of unknown origin: the importance of diagnosing large vessel vasculitis. Q J Nucl Med Mol Imaging 2009;53:51-63.

29 Jenkins I, Vinetz J. The devil is in the details. J Hosp Med 2009;4:382-6.

30 Nordström D, Knight A, Luukkainen $\mathrm{R}$, et al. Beneficial effect of interleukin 1 inhibition with anakinra in adult-onset Still's disease. An open, randomized, multicenter study. J Rheumatol 2012;39:2008-11. 